\title{
Curvature Calculations with Spacetime Algebra
}

\author{
David Hestenes
}

\begin{abstract}
A new method for calculating the curvature tensor is developed and applied to the Scharzschild case. The method employs Clifford algebra and has definite advantages over conventional methods using differential forms or tensor analysis.
\end{abstract}

\section{INTRODUCTION}

Spacetime algebra is a Clifford algebra representing the directional and metrical properties of spacetime. It was originally introduced (Hestenes, 1966) as a unified mathematical language for physics, with applications to electrodynamics, quantum mechanics, and gravitation. It has since been generalized and developed into a comprehensive geometric calculus (GC) (Hestenes and Sobczyk, 1984) with a wide range of computational capabilities. This paper shows how computational problems in gravitation theory can be simplified with GC. Specifically, it presents an efficient method for computing the curvature tensor from a given metric tensor, and illustrates the method by explicit computations from the Schwarzschild metric.

This method should be compared with the method of differential forms presented by Misner et al. (1973) in Chapter 14 of their book. They correctly point out that their method is computationally more efficient than the standard method of tensor analysis. But we shall see that unique features of GC provide even greater efficiency. Moreover, a subsequent paper will show that unlike the method of differential forms, the present method applies directly to the computation of gravitational precession for quantum mechanical as well as classical particles.

The present computational method is a straightforward application of the general method of fiducial frames developed in Chapter 6 of Hestenes and Sobczyk (1984). I will employ their results without repeating the derivations. But first I review the basic definitions of GC with slight changes in notation appropriate for the application to spacetime.

\section{FRAMES}

We represent spacetime by a four-dimensional vector manifold, as defined in Hestenes and Sobczyk (1984). Let $x=x\left(x^{0}, x^{1}, x^{2}, x^{3}\right)$ be a spacetime point parametrized by coordinates $x^{\mu}$, where $\mu=0,1,2,3$. A coordinate frame $\left\{\mathrm{g}_{\mu}=\mathrm{g}_{\mu}(x)\right\}$ at each point $x$ is defined by the partial derivatives,

$$
\mathrm{g}_{\mu}=\partial_{\mu} x
$$

The components of the metric tensor are given by the inner products

$$
\mathrm{g}_{\mu \nu}=\mathrm{g}_{\mu} \cdot \mathrm{g}_{\nu}
$$


A reciprocal coordinate frame $\left\{\mathrm{g}^{\mu}=\mathrm{g}^{\mu}(x)\right\}$ is defined by the gradients of the coordinate functions $x_{\mu}=x_{\mu}(x)$ :

$$
\begin{gathered}
\mathrm{g}^{\mu}=\square x^{\mu} \\
\mathrm{g}^{\mu} \cdot \mathrm{g}_{\nu}=\mathrm{g}^{\mu \alpha} \mathrm{g}_{\alpha \nu}=\delta^{\mu}{ }_{\nu}
\end{gathered}
$$

where $\mathrm{g}^{\mu \alpha}=\mathrm{g}^{\mu} \cdot \mathrm{g}^{\alpha}$.

The coordinate frame $\left\{\mathrm{g}_{\mu}\right\}$ is related to a fiducial frame $\left\{\gamma_{\mu}\right\}$ by a fiducial tensor $h$. The fiducial frame is orthonormal, so

$$
\gamma_{\mu} \cdot \gamma_{\nu}=\eta_{\mu} \delta_{\mu \nu}
$$

where $\eta_{\mu}=\gamma_{\mu}^{2}$ is the signature indicator. Its reciprocal frame $\left\{\gamma^{\mu}\right\}$ is given by

$$
\gamma^{\mu}=\eta_{\mu} \gamma_{\mu}
$$

so $\gamma^{\mu} \cdot \gamma_{\nu}=\delta_{\nu}^{\mu}$. We may assume that both coordinate frame and fiducial frame are righthanded, so

$$
i=\gamma_{0} \gamma_{1} \gamma_{2} \gamma_{3}
$$

where $i=i(x)$ is the unit right-handed pseudoscalar for the spacetime manifold.

The fiducial tensor $h$ is a symmetric tensor relating the coordinate and fiducial frames by the linear transformation

$$
\begin{gathered}
\mathrm{g}_{\mu}=h\left(\gamma_{\mu}\right)=h^{\nu}{ }_{\mu} \gamma_{\nu} \\
\gamma^{\mu}=h\left(\mathrm{~g}^{\mu}\right)=h^{\mu}{ }_{\nu} \mathrm{g}^{\nu}=h^{\mu}{ }_{\nu} \square x^{\nu}
\end{gathered}
$$

The matrix elements of the fiducial tensor are

$$
h^{\mu}{ }_{\nu} \equiv \gamma^{\mu} \cdot \mathrm{g}_{\nu}=\gamma^{\mu} \cdot h\left(\gamma_{\nu}\right)=h\left(\gamma^{\mu}\right) \cdot \gamma_{\nu}=\mathrm{g}^{\mu} \cdot \gamma_{\nu}
$$

The fiducial tensor is related to the metric tensor by

$$
\mathrm{g}_{\mu \nu}=\mathrm{g}_{\mu} \cdot \mathrm{g}_{\nu}=h\left(\gamma_{\mu}\right) \cdot h\left(\gamma_{\nu}\right)=h_{\mu}^{\alpha} \eta_{\alpha} h_{\nu}^{\alpha}
$$

The symmetry of the fiducial tensor allows us to write this in the form

$$
\mathrm{g}_{\mu \nu}=\gamma_{\mu} \cdot h^{2}\left(\gamma_{\nu}\right) \equiv \gamma_{\mu} \cdot \mathrm{g}\left(\gamma_{\nu}\right)
$$

Thus, we can regard the metric tensor as a linear transformation $\mathrm{g}=h^{2}$ on the fiducial frame, so the fiducial tensor $h=\mathrm{g}^{\frac{1}{2}}$ is a square root of the metric tensor. This suggests that, with suitable provisos, the fiducial tensor can be interpreted as a gravitational strain tensor describing a distortion of flat space. But that theme will not be pursued here. I will be content to note that for curvature computations the fiducial tensor is simpler than the metric tensor. 


\section{DERIVATIVES}

According to Hestenes and Sobczyk (1984), the fundamental differential operator on a vector manifold is the derivative $\partial=\partial_{x}$ with respect to a point $x$ on the manifold. All other differential operators can be expressed as algebraic functions of this operator. In particular, coordinate derivatives are related to $\partial$ by

$$
\mathrm{g}_{\mu} \cdot \partial=h_{\mu}^{\nu} \gamma_{\nu} \cdot \partial \equiv \partial / \partial x^{\mu}
$$

Although it is best to define $\partial$ independently of coordinates, as done in Hestenes and Sobczyk (1984), if the manifold (or some part of it) is parametrized by coordinates, the point derivative $\partial$ can be obtained from the coordinate derivatives by

$$
\partial=g^{\mu} \partial_{\mu}
$$

For the directional coderivative we adopt the notation

$$
\square_{\mu}=\mathrm{g}_{\mu} \cdot \square=h_{\mu}^{\nu} \gamma_{\nu} \cdot \square
$$

This differential operator is equivalent to the conventional covariant derivative. It is related to the point coderivative $\square$ by

$$
\square=\mathrm{g}^{\mu} \square \mu
$$

The coderivative $\square$ is defined in terms of the derivative $\partial$ in Hestenes and Sobczyk (1984). But it can be defined alternatively by specifying the coderivatives of a fiducial frame, as is done below.

Since a fiducial frame is orthonormal, it can only rotate when it is displaced along a curve in the manifold. Therefore, the directional coderivatives of the fiducial vectors must have the form

$$
\square_{\mu} \gamma_{\nu}=\omega_{\mu} \cdot \gamma_{\nu}
$$

where $\omega_{\mu}$ is the "angular velocity" of the rotation for a displacement in the $g_{\mu}$ direction. The four $\omega_{\mu}$ are bivectors determining a Riemannian connection for the manifold.

To describe the coderivative of any differentiable multivector field $A=A(x)$, i.e., any function with values in the spacetime algebra, it is convenient to introduce the fiducial derivative $d_{\mu}$ defined by

$$
d_{\mu} \gamma_{\nu}=0
$$

and

$$
d_{\mu} \phi=\partial_{\mu} \phi
$$

for any scalar-valued function $\phi=\phi(x)$. Then we can write

$$
\square_{\mu} A=d_{\mu} A+\omega_{\mu} \times A
$$

where $B \times A \equiv(1 / 2)(B A-A B)$ is the commutator product. The commutator of the coderivatives gives

$$
\left[\square_{\mu}, \square_{\nu}\right] A=\omega_{\mu \nu} \times A
$$


where

$$
\omega_{\mu \nu}=d_{\mu} \omega_{\nu}-d_{\nu} \omega_{\mu}+\omega_{\mu} \times \omega_{\nu}=R\left(\mathrm{~g}_{\mu} \wedge \mathrm{g}_{\nu}\right)
$$

is the curvature tensor evaluated on the bivector $\mathrm{g}_{\mu} \wedge \mathrm{g}_{\nu}$. By virtue of (18a) and (18b), the fiducial derivatives in (21) can be computed from

$$
d_{\mu} \omega_{\nu}=\frac{1}{2}\left(\partial_{\mu} \omega_{\nu}^{\alpha \beta}\right) \gamma_{\alpha} \wedge \gamma_{\beta}
$$

where $\omega_{\nu}^{\alpha \beta}=\gamma^{\alpha} \cdot \omega_{\nu} \cdot \gamma^{\beta}=\omega_{\nu} \cdot\left(\gamma^{\beta} \wedge \gamma^{\alpha}\right)$.

The curvature tensor $R(B)$ is a linear symmetric bivector-valued function of a bivector variable. By virtue of its linearity, (8) in (21) yields

$$
\omega_{\mu \nu}=h_{\mu}{ }^{\alpha} h_{\nu}{ }^{\beta} \omega_{\alpha \beta}
$$

where

$$
\omega_{\alpha \beta}=R\left(\gamma_{\alpha} \wedge \gamma_{\beta}\right)
$$

is the curvature tensor evaluated on the fiducial bivector $\gamma_{\alpha} \wedge \gamma_{\beta}$. The covariant tensor components of the curvature tensor are given by

$$
R_{\mu \nu \alpha \beta}=R\left(\mathrm{~g}_{\mu} \wedge \mathrm{g}_{\nu}\right) \cdot\left(\mathrm{g}_{\alpha} \wedge \mathrm{g}_{\beta}\right)=\omega_{\mu \nu} \cdot\left(\mathrm{g}_{\alpha} \wedge \mathrm{g}_{\beta}\right)=\left(\mathrm{g}_{\mu} \wedge \mathrm{g}_{\nu}\right) \cdot \omega_{\alpha \beta}
$$

\section{CURVATURE CALCULATIONS}

As shown in Hestenes and Sobczyk (1984), (17) can be solved for the connection bivectors $\omega_{\mu}$, with the result

$$
\omega_{\mu}=\frac{1}{2}\left(\gamma_{\alpha} \wedge \square \wedge \gamma^{\alpha}\right) \cdot \mathrm{g}_{\mu}-h_{\mu}^{\nu} \square \wedge \gamma_{\nu}
$$

and the cocurls can be obtained from the fiducial tensor with

$$
\wedge \gamma_{\mu}=\eta_{\mu}\left(\square h_{\nu}^{\mu}\right) \wedge \mathrm{g}^{\nu}
$$

where $\square h^{\mu}{ }_{\nu}=\partial h^{\mu}{ }_{\nu}$ is the gradient of the scalar-valued $h^{\mu}{ }_{\nu}$.

One can calculate the curvature tensor from the fiducial tensor by using (26), (25), and (21) to determine the following quantities in sequence:

$$
h_{\nu}^{\mu} \rightarrow \square \wedge \gamma_{\mu} \rightarrow \omega_{\mu} \rightarrow \omega_{\mu \nu}
$$

For orthogonal coordinates, the $\gamma_{\mu}$ are eigenvectors of the fiducial tensor, and one has the simplifications

$$
\begin{aligned}
h_{\nu}^{\mu} & =h_{\nu} \delta_{\nu}^{\mu} \\
\gamma_{\mu} & =\eta_{\mu} \gamma^{\mu}=\eta_{\mu} h_{\mu} \square x^{\mu}=h_{\mu}^{-1} g_{\mu} \\
\omega_{\mu} & =-h_{\mu} \square \wedge \gamma_{\mu}=\gamma_{\mu} \wedge \square h_{\mu}=\gamma_{\mu} \wedge \gamma^{\alpha} h_{\alpha}^{-1} \partial_{\alpha} h_{\mu} \\
d_{\mu} \omega_{\nu} & =\left[\eta_{\alpha} \partial_{\mu}\left(h_{\alpha}^{-1} \partial_{\alpha} h_{\nu}\right)\right] \gamma_{\nu} \wedge \gamma_{\alpha}
\end{aligned}
$$


Conventional calculations of curvature begin specifying the metric tensor by writing the "line element"

$$
d x^{2}=d x \cdot d x=\mathrm{g}_{\mu \nu} d x^{\mu} d x^{\nu}
$$

with the $g_{\mu \nu}$ expressed as defined functions of coordinates $x^{\mu}$. We can determine the fiducial tensor from (32) by using (11), so we can proceed from the conventional starting point to calculate the curvature by the method of fiducial frames.

As an example of fundamental importance, one cannot do better than calculate the curvature tensor from the Schwarzschild line element

$$
\begin{aligned}
d x^{2} & =e^{2 \Phi} d t^{2}-e^{2 \lambda} d r^{2}-r^{2}\left(d \theta^{2}+\sin ^{2} \theta d \phi^{2}\right) \\
& =h_{t}^{2} d t^{2}-h_{r}^{2} d r^{2}-h_{\theta}^{2} d \theta^{2}-h_{\phi}^{2} d \phi^{2}
\end{aligned}
$$

where $\Phi=\Phi(r, t)$ and $\lambda=\lambda(r, t)$ are scalar functions independent of coordinates $\theta$ and $\phi$. Comparing (33) with (29), one can immediately write down

$$
\begin{aligned}
\gamma_{t} & =e^{\Phi} \square t & & h_{t}=e^{\Phi} \\
\gamma_{r} & =-e^{\lambda} \square r & & h_{r}=e^{\lambda} \\
\gamma_{\theta} & =-r \square \theta & & h_{\theta}=r \\
\gamma_{\phi} & =-r \sin \theta \square \phi & & h_{\phi}=r \sin \theta
\end{aligned}
$$

The use of the same symbols $t, r, \theta, \phi$ for indices, coordinates, and independent variables should not cause confusion, as the distinction is clear from the context.

Using (34) in (30), one obtains easily

$$
\begin{aligned}
& \omega_{t}=-\gamma_{t} \wedge \gamma_{r} \Phi_{r} e^{\Phi-\lambda} \\
& \omega_{r}=\gamma_{r} \wedge \gamma_{t} \lambda_{t} e^{\lambda-\Phi} \\
& \omega_{\theta}=-\gamma_{\theta} \wedge \gamma_{r} e^{-\lambda} \\
& \omega_{\phi}=-\gamma_{\phi} \wedge\left(\gamma_{r} e^{-\lambda} \sin \theta+\gamma_{\theta} \cos \theta\right)
\end{aligned}
$$

where subscripts on $\Phi$ and $\lambda$ indicate derivatives, that is, $\Phi_{r}=\partial_{r} \Phi$ and $\lambda_{t}=\partial_{t} \lambda$.

The simple computation of $\omega_{\mu}$ in (35) may be compared with the corresponding computation from the Friedmann metric in Misner et al. (1973, pp. 356). Skillful guessing with differential forms is advocated by Misner et al., while we merely apply equation (30), which has no counterpart in Misner et al. Indeed, application of (30) is easier as well as more straightforward than guessing, because the effort needed to check each guess has been expended once and for all in the derivation of (30).

It should be noted that the "wedges" in (35) are actually unnecessary because the $\gamma_{\mu}$ are orthogonal. This greatly simplifies the evaluation of commutators $\omega_{\mu} \times \omega_{\nu}$, and one easily determines that the nonvanishing commutators have the values

$$
\begin{aligned}
& \omega_{t} \times \omega_{\theta}=\gamma_{t} \wedge \gamma_{\theta} \Phi_{r} e^{\Phi-2 \lambda} \\
& \omega_{t} \times \omega_{\phi}=\gamma_{t} \wedge \gamma_{\phi} \Phi_{r} e^{\Phi-2 \lambda} \sin \theta \\
& \omega_{r} \times \omega_{\theta}=\gamma_{\theta} \wedge \gamma_{t} \lambda_{t} e^{-\Phi} \\
& \omega_{r} \times \omega_{\phi}=\gamma_{\phi} \wedge \gamma_{t} \lambda_{t} e^{-\Phi} \sin \theta \\
& \omega_{\theta} \times \omega_{\phi}=\left(\gamma_{r} e^{-\lambda} \cos \theta-\gamma_{\theta} e^{-2 \lambda} \sin \theta\right) \wedge \gamma_{\phi}
\end{aligned}
$$


Using (31) to compute the relevant fiducial derivatives from (35), one finds that the nonvanishing terms are

$$
\begin{aligned}
d_{r} \omega_{t} & =-\gamma_{t} \wedge \gamma_{r}\left(\Phi_{r r}+\Phi_{r}^{2}-\Phi_{r} \lambda_{r}\right) e^{\Phi-\lambda} \\
d_{t} \omega_{r} & =\gamma_{r} \wedge \gamma_{t}\left(\lambda_{t t}+\lambda_{t}^{2}-\lambda_{t} \Phi_{t}\right) e^{\lambda-\Phi} \\
d_{t} \omega_{\theta} & =\gamma_{\theta} \wedge \gamma_{r} \lambda_{t} e^{-\lambda} \\
d_{t} \omega_{\phi} & =\gamma_{\phi} \wedge \gamma_{r} \lambda_{t} e^{-\lambda} \sin \theta \\
d_{r} \omega_{\theta} & =\gamma_{\theta} \wedge \gamma_{r} \lambda_{r} e^{-\lambda} \\
d_{r} \omega_{\phi} & =\gamma_{\phi} \wedge \gamma_{r} \lambda_{r} e^{-\lambda} \sin \theta \\
d_{\theta} \omega_{\phi} & =\left(\gamma_{r} e^{-\lambda} \cos \theta-\gamma_{\theta} \sin \theta\right) \wedge \gamma_{\phi}
\end{aligned}
$$

Finally, one obtains the curvature bivectors by inserting (36) and (37) into (21). I display the result to show both the coordinate components and the fiducial components $\omega_{\hat{\mu} \hat{\nu}} \equiv R\left(\gamma_{\mu} \wedge \gamma_{\nu}\right)$

$$
\begin{aligned}
\omega_{t r} & =e^{\Phi+\lambda} \omega_{\hat{t} \hat{r}} \\
& =e^{\Phi+\lambda} \gamma_{r} \wedge \gamma_{t}\left[\left(\lambda_{t t}+\lambda_{t}^{2}-\lambda_{t} \Phi_{t}\right) e^{-2 \Phi}-\left(\Phi_{r r}+\Phi_{r}^{2}-\Phi_{r} \lambda_{r}\right) e^{-2 \lambda_{r}}\right] \\
\omega_{t \theta} & =e^{\Phi} r \omega_{\hat{t} \hat{\theta}} \\
& =e^{\Phi} r \gamma_{\theta} \wedge\left[\gamma_{r} \lambda_{t} r^{-1} e^{-\Phi-\lambda}+\lambda_{t} \Phi_{r} r^{-1} e^{-2 \lambda}\right] \\
\omega_{t \phi} & =e^{\Phi} r \sin \theta \omega_{\hat{t} \hat{\phi}} \\
& =e^{\Phi} r \sin \theta \gamma_{\phi} \wedge\left[\gamma_{r} \lambda_{t} r^{-1} e^{-\Phi-\lambda}+\lambda_{t} \Phi_{r} r^{-1} e^{-2 \lambda}\right] \\
\omega_{r \theta} & =e^{\lambda} r \omega_{\hat{r} \hat{\theta}} \\
& =e^{\lambda} r \gamma_{\theta} \wedge\left[\gamma_{r} \lambda_{r} r^{-1} e^{-2 \lambda}-\gamma_{t} \lambda_{t} r^{-1} e^{-\Phi-\lambda}\right] \\
\omega_{r \phi} & =e^{\lambda} r \sin \theta \omega_{\hat{r} \hat{\phi}} \\
& =e^{\lambda} r \sin \theta \gamma_{\phi} \wedge\left[\gamma_{r} \lambda_{r} r^{-1} e^{-2 \lambda}-\gamma_{t} \lambda_{t} r^{-1} e^{-\Phi-\lambda}\right] \\
\omega_{\theta \phi} & =r^{2} \sin \theta \omega_{\hat{\theta} \hat{\phi}} \\
& =r^{2} \sin \theta \gamma_{\theta} \wedge \gamma_{\phi} r^{-2}\left(e^{-2 \lambda}-1\right)
\end{aligned}
$$

Of course, if we had so desired, we could have computed any one of these bivectors independently of the others. Also, many of the coefficients in (38) are equivalent because of the symmetry property of curvature, $A \cdot R(B)=B \cdot R(A)$. This redundancy provides a check on the computations.

The orthogonality of the $\gamma^{\mu}$ makes it especially easy to contract (38) to get the Ricci tensor

$$
R\left(\gamma_{\mu}\right)=\gamma^{\nu} \cdot R\left(\gamma_{\nu} \wedge \gamma_{\mu}\right)=\gamma^{\nu} \cdot \omega_{\hat{\nu} \hat{\mu}}
$$

We obtain

$$
\begin{aligned}
R\left(\gamma_{t}\right)=2 \gamma_{r} \lambda_{t} r^{-1} e^{-\Phi-\lambda}+\gamma_{t}[ & \left(\lambda_{t t}+\lambda_{t}^{2}-\lambda_{t} \Phi_{t}\right) e^{-2 \Phi} \\
& \left.-\left(\Phi_{r r}+\Phi_{r}^{2}-\Phi_{r} \lambda_{r}-2 \Phi_{r} r^{-1}\right) e^{-2 \lambda}\right]
\end{aligned}
$$




$$
\begin{aligned}
R\left(\gamma_{r}\right)=2 \gamma_{r} \lambda_{t} r^{-1} e^{-\Phi-\lambda}+\gamma_{r}[ & -\left(\lambda_{t t}+\lambda_{t}^{2}-\lambda_{t} \Phi_{t}\right) e^{-2 \Phi} \\
& \left.+\left(\Phi_{r r}+\Phi_{r}^{2}-\Phi_{r} \lambda_{r}+2 \lambda_{r} r^{-1}\right) e^{-2 \lambda}\right] \\
R\left(\gamma_{\theta}\right)=\gamma_{\theta}\left[\left(\lambda_{t}-\Phi_{r}\right) r^{-1} e^{-2 \lambda}+\right. & \left.\left(1-e^{-2 \lambda}\right) r^{-2}\right]=\gamma_{\phi} \gamma_{\theta} R\left(\gamma_{\phi}\right)
\end{aligned}
$$

Setting $R\left(\gamma_{\mu}\right)=0$ to find the "free space" gravitational field according to Einstein's theory, we see immediately that (39a) and (39b) imply that $\Phi_{t}=\lambda_{t}=0$ and $\Phi=-\lambda$, so (39c) reduces to the equation

$$
r \lambda_{r}+e^{-\lambda}-1=0
$$

This integrates to the famous Schwarzschild solution

$$
e^{-\lambda}=1-\frac{2 \kappa}{r}=e^{\Phi}
$$

where $\kappa$ is a constant.

We can substitute (40) into (35) to get the $\omega_{\mu}$ as an explicit function of the coordinates. The result can be used to describe the motion of a test body, as explained in a subsequent paper.

\section{REFERENCES}

Hestenes, D. (1966). Space-Time Algebra, Gordon and Breach, New York.

Hestenes, D., and Sobczyk, G. (1984). Clifford Algebra to Geometric Calculus, Reidel, Dordrecht.

Misner, C., Thorne, K., and Wheeler, J. (1973). Gravitation, Freeman, San Francisco. 